\title{
Séquelles cognitives, physiques et psychopathologiques à long terme de multiples commotions cérébrales : illustration par l'évaluation et la prise en charge d'un hockeyeur semi- professionnel
}

\author{
Catia Beni ${ }^{1}$ \\ ${ }^{1}$ Département des neurosciences et sciences du mouvement. Université de Fribourg, Suisse.
}

\begin{abstract}
Dans la pratique du hockey sur glace, les commotions cérébrales à répétition sont malheureusement fréquentes et provoquent une cascade de conséquences, qu'elles soient scolaires ou professionnelles (c.-à-d., arrêts, absences), sportives (c.-à-d., baisse des performances, fin de carrière) ou médicales (p. ex., présence de symptômes à long terme). Il existe très peu d'articles qui documentent de manière clinique les prises en charge et les séquelles cognitives, physiques et psychopathologiques de commotions cérébrales à répétition. L'objectif de ce chapitre est double. Tout d'abord, après avoir fourni un peu de théorie des connaissances actuelles sur les commotions cérébrales, les éléments de la prise en charge qui ont été entrepris avec un hockeyeur semi-professionnel ayant subi de nombreuses commotions cérébrales seront exposés, à savoir l'aide dans la diminution des symptômes post-commotionnels et dans l'acceptation de mettre un terme à la carrière de hockeyeur. Deuxièmement, il montrera les performances cognitives de cet athlète sportif, 11 mois après sa dernière commotion cérébrale.
\end{abstract}

Mots-clés : commotion cérébrale à répétition, hockey sur glace, séquelles à long terme, bilan neuropsychologique, prise en charge cognitive comportementale

\begin{abstract}
In the practice of ice hockey, recurrent concussions are unfortunately frequent and cause a cascade of consequences, whether they are academic or professional (i.e., stops, absences), sportive (i.e., decrease in performances, end of career) or medical (e.g., long-term symptoms). In the literature, there are very few articles that clinically document the cognitive, behavioral and emotional management and sequelae of recurrent concussions. The purpose of this chapter is twofold. First, after providing a little theory of current knowledge about concussions, the elements of management that has been undertaken with a semiprofessional hockey player,who has suffered many concussions will be exposed, namely the help in the reduction of post-concussive symptoms and the help provided in the acceptance to put an end to the hockey career. Second, it will show the cognitive performance of this sportsman, 11 months after his latest concussion.
\end{abstract}

Keywords: recurrent concussion, ice hockey, long-term sequelae, neuropsychological assessment, cognitivebehavioral management

\section{Définition du sujet de recherche}

Les commotions cérébrales $(\mathrm{CC})$ dans le sport touchent de nombreux sportifs, que ce soit des enfants, des adolescents ou des adultes, des professionnels ou des amateurs, en match ou en entraînement (Harmon et al., 2013). Depuis quelques années maintenant, les CC sont au centre des préoccupations médicales dans le sport, notamment en raison d'une plus forte sensibilisation à la possibilité de séquelles à long terme.

Correspondence concerning this article should be adressed to/ La correspondance concernant cet article doit être adressée à :

Dr Psych Catia Beni, Neuropsychologue, Département des neurosciences et sciences du mouvement, Université de Fribourg. Email/courriel: catia.beni@fsp-hin.ch
Une CC est un traumatisme crânio-cérébral léger. Le mot CC est surtout employé dans le contexte du sport, mais le terme médical est traumatisme crânien léger (TCL). C'est un processus physiopathologique complexe touchant le cerveau, qui entraîne rapidement une brève altération de la fonction neurologique (McCrory et al., 2017) ; à la suite d'un coup direct donné à la tête, au visage, à la nuque; ou à la suite d'un coup au corps dont la force se répercute jusqu'à la tête et entraine un mouvement rapide du cerveau. À cela peuvent s'ajouter des mécanismes de coup - contre coup, mais aussi des mécanismes rotatoires (Purcell, 2014). Comme c'est une atteinte fonctionnelle du cerveau et non une atteinte structurelle, $79 \%$ des scanners cérébraux et $75 \%$ des imageries par résonnance magnétique (IRM) cérébrales ne montrent aucune lésion (Ellis et al., 2015). Les méthodes plus modernes comme l'imagerie du tenseur de diffusion apportent de meilleures informations, mais elles ne sont aujourd'hui utilisées qu'en recherche 
(Shenton et al., 2018). Une CC peut survenir dans la pratique sportive, mais également dans n'importe quel contexte de vie (p. ex., accident domestique ou accident de la voie publique). Certains sports sont naturellement plus à risque que d'autres de séquelles à long terme, comme le hockey sur glace, la boxe, le football américain et le rugby (Harmon et al., 2013).

$\mathrm{Au}$ lieu de parler de $\mathrm{CC}$ légère ou $\mathrm{CC}$ sévère, termes souvent liés à la gravité du choc et/ou à la présence d'une perte de connaissance, il est aujourd'hui plus juste d'évoquer la CC sous un angle de séquelles et de durée de récupération, car même des chocs légers peuvent parfois déclencher des conséquences majeures. Dans ce contexte, les CC dites simples représentent 80 à $90 \%$ des CC diagnostiquées. Elles se résolvent généralement dans les 7 à 10 jours, au maximum dans le mois, avec une certaine hétérogénéité (Castile et al., 2012). Puis, les CC dites complexes ou à évolution lente sont une petite minorité de CC (c.-à-d., 10 à 15\%). Des séquelles persistent au-delà de 7 à 10 jours. Le blessé peut présenter alors un syndrome post-commotionnel (SPC). Selon la définition de la CIM-10 (2009) sous le code F07.2, le SPC correspond à des symptômes physiques, cognitifs et psychologiques, associés à des déficits cognitifs consécutifs persistants, à la suite d'un traumatisme crânien. Ces symptômes peuvent perturber le fonctionnement social, familial, professionnel et/ou scolaire de la personne au quotidien. Dans certains cas, cela implique la nécessité de stopper l'activité sportive, mais aussi l'activité scolaire ou professionnelle (Castile et al., 2012). Tator et ses collaborateurs (2016) ont montré que lors d'un SPC, les séquelles à long terme ont une médiane à sept mois, mais peuvent dans certains cas perdurer à vie. Ces auteurs ont également montré que ce syndrome se diagnostique normalement après plusieurs $\mathrm{CC}$, mais dans $23,1 \%$, un SPC survient après la première $\mathrm{CC}$. Le nombre moyen de symptômes persistants est de 8,1. Lors de séquelles plus importantes, le syndrome du second impact (c.-à-d., un gonflement cérébral diffus ; McCrory et al., 2012) ou l'encéphalopathie chronique traumatique sont des entités médicales qui apportent des séquelles majeures et handicapantes et sont une préoccupation majeure (Manley et al., 2017; Mez et al., 2017).

La gestion des CC et des séquelles est ici le cœur du problème. De nombreuses fédérations sportives ont établi un protocole strict de retour au sport afin de gérer la récupération post-commotionnelle et diminuer le risque de séquelles à long terme. Ce protocole est globalement efficace dans le cadre des CC simples, car après un temps de repos de 24 à 48 heures, il propose une reprise graduelle des activités en tenant compte de la présence ou non de symptômes. En médecine du sport, pour évaluer ces symptômes, le Grade Symptom Checklist (GSC), la seconde partie de l'outil Sport Concussion Assesment Tool, est largement utilisé. Il est composé de 22 questions autoévaluatives, évaluées par une échelle de Likert en sept points et qui répertorie les symptômes post-commotionnels classiques en lien avec une atteinte cervicale, une atteinte vestibulo-oculaire, une atteinte cognitive, des manifestations anxio-dépressives ou comportementales ou encore des troubles du sommeil. Ces symptômes sont : le mal de tête, la pression dans le crâne, les douleurs au cou, les nausées, l'étourdissement, la vision trouble, les problèmes d'équilibre, l'hypersensibilité au bruit ou à la lumière, la sensation d'être ralenti, d'être dans le brouillard, le sentiment d'anomalie, le manque de concentration, les troubles de mémoire, la confusion, l'émotivité accrue, l'irritabilité, la tristesse, la nervosité, les difficultés d'endormissement, la fatigue et la somnolence. Dès lors que le score au GSC est plus petit que 15 (en moyenne, car le score varie selon l'âge et le sexe ; ImPACT Applications, 2020), une planification des activités est établie, en précisant la durée, la fréquence et l'intensité de chaque activité (Howell et al., 2018). Puis, le Sport Concussion in Sport Group a établi en 2001 un protocole de retour au jeu en six étapes (McCrory et al., 2017). Il permet une reprise graduelle de l'activité sportive, allant du repos à la reprise complète de la compétition. Cette méthode est efficace pour un sportif qui présente une $\mathrm{CC}$ à évolution simple ou sans antécédent de CC, mais elle est clairement insuffisante pour des CC à évolution lente ou lors de multiples CC.

Il n'existe que peu de documents dans la littérature qui présente les prises en charge de ces problématiques complexes. En outre, il a été démontré que $49 \%$ des médecins de famille, $52 \%$ des médecins d'urgence et $27 \%$ des pédiatres ont déclaré n'avoir aucune idée des critères consensuels concernant la gestion des CC (Stoller et al., 2014). Enfin, les médecins des centres d'urgences dispensent quelques conseils classiques généraux, mais ces derniers ne sont clairement pas suffisants. En revanche, plus récemment, Beni (2019) présente la prise en charge d'un jeune joueur de rugby commotionné. Elle y propose plusieurs pistes pratico-pratiques de prises en charge individualisées, en mentionnant par exemple quelques règles d'or dans cette prise en charge (Beni, 2019). Ces règles d'or peuvent se résumer ainsi (a) être un patient patient, (b) faire peu, se reposer beaucoup, (c) maximiser ce qui fait du bien, minimiser ce qui fait du mal, (d) la blessure est une opportunité, (e) l'intensité ou durée ou fréquence, (f) s'arrêter avant, (g) tout fatigue, (h) ne rien faire, c'est prendre soin de soi, (i) éviter ou bannir les écrans et (j) être bien entouré. Ces règles d'or sont travaillées et explicitées en fonction des besoins de la personne. Les explications doivent être claires et détaillées et il peut être utile de s'aider de dessins ou de schémas. Cela permet de favoriser la compréhension, d'augmenter le niveau de concentration et de varier les sources d'encodage d'une information, principe capital de la rééducation en neuropsychologie.

\section{Revue de la littérature}

Pendant longtemps, le diagnostic de $\mathrm{CC}$ était posé uniquement si une perte de connaissance avait lieu. Aujourd'hui, des études montrent que seuls 5 à $9 \%$ des athlètes perdent connaissance lors d'une CC (Castile et al., 2012). Malheureusement, encore trop fréquemment, et cela même dans les centres d'urgence, la gravité ou même le diagnostic n'est posé que s'il y a perte de connaissance. Cela est regrettable, car il a été démontré que l'amnésie circonstancielle est un meilleur indicateur de gravité ou de la présence d'une $\mathrm{CC}$, que la perte de connaissance : plus l'amnésie circonstancielle est longue, plus les séquelles sont importantes et la récupération plus longue (Tator et al., 2016). 
Les séquelles à la suite d'une CC peuvent être multiples, variées et différentes pour chaque individu et pour chaque CC. Il existe des séquelles à court, moyen et long terme. À court et moyen terme, les études sont majoritairement en accord sur la présence de séquelles, bien que certaines études plaident l'absence de séquelles. Ces études sont discutables sur le plan de la méthodologie employée (p. ex., échantillon petit ou hétérogène, délai depuis la commotion non contrôlée, études se basant sur le test Immediate PostConcussion Assessment and Cognitive Testing pour établir la présence de troubles ; Brooks et al., 2016). Quatre types de problèmes sont classiquement évoqués : 1) les symptômes physiques (p. ex., maux de tête, nausées, vertiges, troubles visuels, photo et phono phobie, perte de conscience, perte d'équilibre), 2) les changements de comportement (p. ex., irritabilité, labilité émotionnelle, tristesse, anxiété et émotions inadéquates), 3) les troubles cognitifs (p. ex., ralentissement, difficultés de concentration et de mémoire), 4) les troubles du sommeil (p.ex., somnolence, difficultés d'endormissement, hyper ou hyposomnie, insomnie ; Purcell, 2014 ; McCrory et al., 2017). Ces séquelles peuvent subsister à court, moyen et long terme (Tator et al., 2016). Les trois séquelles les plus fréquemment reportées par les sportifs sont les maux de tête $(85,5 \%)$, les étourdissements $(64,4 \%)$ et les troubles de la concentration (47,8\% ; Castile et al., 2012). La perte de connaissance n'est mentionnée que dans $10 \%$ des cas (Mansell et al., 2010). Les séquelles sont variées et une prise en charge adéquate est donc nécessaire.

\section{Objectifs}

Les séquelles à long terme des $\mathrm{CC}$ sont une forte préoccupation pour les neuropsychologues et pour les professionnels de la santé qui travaillent dans le milieu du sport de contact. En effet, ces séquelles restent encore peu prises en charge, notamment pour des raisons financières de la part de certaines fédérations sportives, mais aussi de la part des assurances. Naturellement, tout cela questionne sur les aspects éthiques à vouloir banaliser ou minimiser les conséquences, très souvent invalidantes (Gillett, 2018).

Les objectifs de ce chapitre sont multiples. La présentation du cas FB permettra tout d'abord de montrer les données de séquelles cognitives, physiques et psychopathologiques à long terme d'un joueur de hockey sur glace, qui a subi de nombreuses CC cérébrales mal soignées. Mais, cela permettra également de donner des éléments de prise en charge des troubles spécifiques de ce sportif, en intégrant à la fois des techniques neuropsychologiques, mais aussi des techniques issues des thérapies cognitivo-comportementales. Cette étude de cas est originale et inédite, car la littérature foisonne d'articles qui analysent des données de groupes sur les séquelles postcommotionnelles, mais que très rarement des données issues d'un cas unique. Cela d'autant plus que cette étude de cas aborde autant la partie de l'évaluation que de la prise en charge. Ensuite, et l'intérêt est encore plus important, il n'existe pas d'articles ou de chapitres traitant de la prise en charge des séquelles post-commotionnelles à long terme. Cette dernière partie est particulièrement utile pour les professionnels engagés dans le milieu du sport, car des conseils, des outils et des méthodes très pratiques et pragmatiques sont proposés, ce qui habituellement fait défaut dans les publications qui ne donnent que des conseils très généraux.

\section{Méthodologie}

\section{Éthique et confidentialité}

FB est venu consulter en cherchant de l'aide et des réponses, à la suite de ses plaintes en lien avec ses nombreuses CC. Très rapidement, il est apparu que sa situation n'était de loin pas inédite et qu'il fallait que sa mauvaise expérience puisse servir à documenter les erreurs de prise en charge, encore très fréquentes dans le milieu du hockey sur glace, mais également dans tous les milieux sportifs à risque de $\mathrm{CC}$. Il lui a été proposé d'écrire son histoire sous un regard neuropsychologique et scientifique et il a, sans hésiter, donné son accord oral et écrit. Il lui a naturellement été garanti l'anonymat et que l'écrit était soumis aux règles éthiques et déontologiques imposées par la Fédération Suisse des Psychologues. Il a lu le contenu de cet article avant sa première soumission et il l'a validé.

\section{Présentation détaillée du cas}

FB est âgé de 29 ans au moment de sa première consultation. C'est un défenseur au hockey sur glace, en ligue mineure, mais qui par le passé a joué dans une ligue professionnelle et aussi dans une équipe nationale. FB est de langue maternelle française et hongroise. Après l'école obligatoire, il s'est inscrit à l'université, mais il a dû arrêter en raison de ses symptômes post-commotionnels. Environ deux à trois ans après, il s'est inscrit à nouveau à l'université en économie et il a obtenu un baccalauréat. Il a également terminé un master en ressources humaines. $\mathrm{Au}$ moment de l'évaluation, il venait de terminer un stage en ressources humaines. Hormis ses troubles en lien avec les $\mathrm{CC}, \mathrm{FB}$ est en bonne santé et ne présente pas d'autres difficultés.

L'historique des différents chocs subis par FB a été répertorié sur la base de ses souvenirs. Le jour J représente la date de sa première consultation. Les dates sont transcrites sur cette base.

\section{Jour J-10 ans}

Lors d'un match, dans une équipe de ligue $\mathrm{A}$, il a été victime d'un traumatisme crânio-cérébral (TCC). Une perte de connaissance, d'une durée indéterminée, est présente, ainsi qu'une amnésie circonstancielle. Son dernier souvenir est environ deux à trois heures avant le choc et son premier souvenir après le choc est dans l'ambulance. Il a été hospitalisé une nuit. Il a eu de la peine à aller mieux et ce n'est que quelques semaines plus tard qu'il a consulté un neurologue, qui lui fait différents examens. Selon ses souvenirs, une imagerie cérébrale a mis en évidence un saignement sur la partie avant du cerveau. Après deux à trois semaines, il a repris le sport, alors qu'il présentait encore de nombreux symptômes (c.-à-d., absences, problèmes de concentration, hypersensibilité à la lumière, importants problèmes de lecture, ralentissement). Sur le plan émotionnel, il était plus triste et il présentait des sautes d'humeur nécessitant des consultations auprès d'un psychiatre. En parallèle, il a vu ses performances chuter au hockey et il a raté la qualification pour les championnats du monde. Sur le plan scolaire, il a arrêté l'université, car il n'arrivait pas à suivre. 


\section{Jour $J-8$ ans}

Nouveau choc lors d'un match. Depuis lors, sa santé physique est très fragile. Il a continué à jouer et la moindre charge le faisait retomber dans les symptômes. Du coup, durant une saison entière, il jouait, prenait un coup et s'arrêtait environ deux semaines, rejouait, etc. Il était lent et il avait des vertiges. Il a fini difficilement ses études de baccalauréat, car il avait de la peine à suivre et il devait travailler énormément en raison des problèmes de concentration et de saccades oculaires.

\section{Entre Jour $J-4$ ans et Jour $J-2$ ans}

Il a arrêté complètement le hockey, ce qui a permis d'apporter une nette diminution des symptômes. En revanche, sur le plan émotionnel, il présentait de nombreuses difficultés, notamment car il n'avait pas été accompagné dans l'arrêt du hockey. Il est tombé alors en forte dépression et il a eu un suivi psychothérapeutique durant plusieurs mois.

\section{Jour J-3 mois}

Il a repris le hockey en ligue mineure et lors d'un match, il a reçu une charge relativement violente à la tête. Il s'est arrêté quelques jours, puis il a repris pour environ 10 matchs, avant de reprendre un choc. Ce dernier choc a nécessité un nouvel arrêt et notre première consultation le jour $\mathrm{J}$.

\section{Jour $J$}

FB consulte en urgence en neuropsychologie quelques semaines après sa énième commotion cérébrale. Une prise en charge a été conduite. Puis, compte tenu de ses nombreuses commotions cérébrales, un bilan neuropsychologique complet à distance de la dernière $\mathrm{CC}$, soit 11 mois plus tard, a été réalisé, afin de mesurer les séquelles à long terme.

\section{Jour $\boldsymbol{J}+8$ mois}

Une consultation auprès d'un médecin psychiatre, conclut que FB souffre d'un Trouble bipolaire type I.

\section{Jour $J+11$ mois}

Un IRM cérébrale a démontré une séquelle hémorragique frontale gauche de $3 \mathrm{~mm}$ sur le plan antéropostérieur.

\section{Prise en charge}

Les aspects de la prise en charge vont être expliqués et détaillés, en fournissant des outils spécifiques à la problématique de FB. Par souci de clarté et de fluidité des propos, il est apparu peu utile de lister ici le contenu des séances en détail, étant donné que certains propos ont été abordés à la première séance, repris à la $3^{\mathrm{e}}$ ou à la $5^{\mathrm{e}}$ séance, etc., cela en fonction des plaintes de FB. Dans toute prise en charge, chaque propos avancé doit être repris lors des séances suivantes, afin de vérifier sa compréhension et son application. Les troubles de mémoire et d'attention étant fréquents à la suite d'un TCL, cela permet aussi de vérifier le risque d'oubli de la discussion ou des conseils apportés et leur compréhension. En revanche, il n'y a pas un guidage

'Si besoin, l'Echelle des Changements de Comportement SocioEmotionnel de Genève peut être administrée. Elle mesure les modifications du comportement après un traumatisme crânien (Beni et al., 2017). strict du déroulement d'une séance, étant donné que le travail se fait sur la base de ce que la personne apporte et que chaque être humain va apporter sa problématique et son histoire de vie. L'aspect individualisé et la vision intégrative d'une prise en charge prennent ici tout leur sens (Van der Linden, 2018). Il est en revanche primordial de fournir lors de la première séance la notion du seuil des symptômes (voir Figure 1). Par expérience, plus le sportif reçoit les bonnes informations de manière précoce à la suite de la blessure, plus la récupération sera positive, car il appliquera dès le départ des stratégies correctes. Le bénéfice substantiel de la psychoéducation dans la récupération est aujourd'hui largement admis; il s'agit d'expliquer les troubles, leur action, leur influence, comment y faire face, etc. (Mittenberg et al., 1996).

\section{Les plaintes et les symptômes}

L'anamnèse reste un point central de toutes prises en charge. Avec FB, plusieurs aspects ont été répertoriés : 1) les facteurs personnels, 2) les aspects cliniques et médicaux de la $\mathrm{CC}, 3)$ les aspects psychologiques, 4) les aspects comportementaux $\left.{ }^{1}, 5\right)$ les antécédents médicaux personnels, 6) l'hygiène de vie, 7) la consommation de substances psychoactives, 8) la situation d'accident, 9) le contexte de l'activité sportive et 10) les facteurs environnementaux (cf. Beni, 2019).

Ensuite, et cela de manière précise, les plaintes actuelles de FB ont été récoltées. Il s'est plaint de vertige à l'effort et de troubles de la concentration au quotidien et pour quasi tout type de tâches mentales. Il a relevé d'importants maux de tête et des troubles du sommeil. Sur demande spécifique, il a évoqué de nombreux oublis au quotidien. Sur le plan visuel, il avait toujours des problèmes de saccades. Il était sous stress permanent, avec un sentiment de mal être général. Il était agité et nerveux. Une évolution négative générale a été constatée, raison pour laquelle il a demandé de l'aide.

Il est alors important de savoir comment ces plaintes ont évoluées, entre le jour de l'accident et le jour de l'anamnèse. En parallèle, une liste des symptômes classiquement présents à la suite d'une $\mathrm{CC}$ est complétée (Annexe A). Ce tableau est nettement plus complet que le GSC et FB l'a rempli en indiquant l'intensité de ses symptômes.

Rapidement FB a posé deux questions, questions récurrentes après un TCL: 1) "Combien de temps cela va prendre ? » et 2) «Comment je peux guérir plus vite ? ». Il faut répondre de manière transparente en expliquant que le délai de récupération n'est pas connu, car il dépend de nombreux paramètres, certains que l'on maîtrise et d'autres pas. Dans ce que le blessé maîtrise, il y a le principe d'adhérence au traitement, c'est-à-dire, plus vite le blessé va comprendre et mettre adéquatement en pratique tout ce qui est expliqué en séance, plus il progressera dans les délais légitimes de récupération. Cela permet aussi de lui faire comprendre qu'il n'y a pas de moyen de «guérir plus vite », comme il le veut, mais par contre en appliquant de mauvaises stratégies ou en adoptant de mauvais comportements, le temps pour récupérer peut être plus long. Dans les éléments sans maîtrise, il y a notamment les événements de vie (p. ex., décès, maladie, pression du management). Dans le principe de transparence, qui est 


\section{SÉQUELLES DE MULTIPLES COMMOTIONS CÉRÉBRALES}

important pour établir une bonne alliance thérapeutique, il faut baliser le parcours de rééducation en expliquant les étapes possibles. Cela montre que si le délai de récupération n'est pas connu, la procédure et les comportements à adopter, pour favoriser cette récupération, sont eux connus.

Ceci fait, il faut ensuite absolument fournir des aides pratico-pratiques en lien avec la problématique personnelle. Dans le cas de FB, deux objectifs sont apparus assez clairement: 1) la gestion des symptômes postcommotionnels et 2) l'accompagnement dans l'arrêt de la pratique du hockey.

\section{La gestion des symptômes post-commotionnels}

En lien avec la problématique de FB, trois points sont au centre des discussions et des aides fournies. Tout d'abord et prioritairement, la notion du seuil des symptômes doit être abordée. Le seuil de symptômes, initialement tiré des propos du professeur Frémont de l'Université de Laval au Québec, se définit comme le niveau au-delà duquel l'intensité ou la durée d'un effort physique ou mental va développer des symptômes post-commotionnels et/ou les augmenter (Figure 1; Beni, 2019; Frémont, 2017). Ensuite, l'importance du repos et du choix des activités doivent également être inculqués rapidement dans la prise en charge, de manière justement à ne pas franchir le seuil des symptômes.

Figure 1

Variation des symptômes en fonction du temps et de l'intensité

1. Augmentation des symptômes lors d'activités physiques ou mentales au-dessus du seuil des symptômes

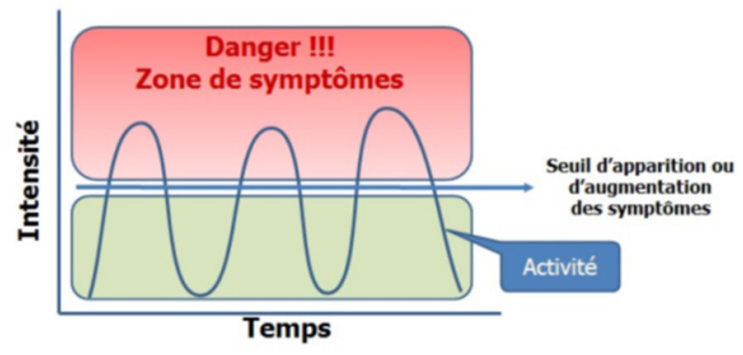

2. Stabilisation de l'état, en respectant le seuil des symptômes

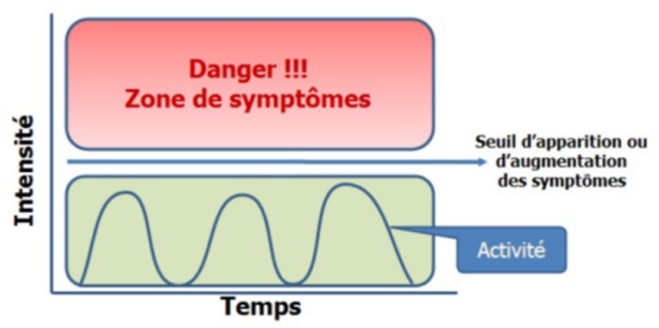

3. Possibilité d'augmenter les activités dans la durée ou dans l'intensité

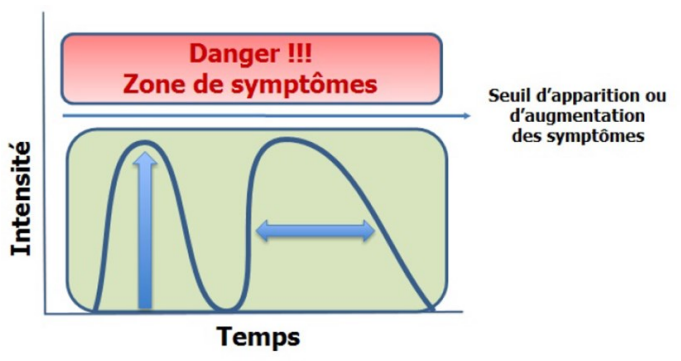


L'importance du repos. Les praticiens conseillent de se reposer, sans plus de précision. Par expérience, le terme se reposer peut prendre des colorations différentes en fonctions de la personne. Certains comprendront une sieste, d'autres lire, d'autres regarder la télévision, d'autres plutôt regarder un mur blanc. Le repos, pour qu'il soit bénéfique dans une récupération post-commotionnelle doit être organisé et donc pleinement expliqué, calculé et mesuré dans ce qui va être fait, quand et comment. Le repos permet au cerveau de «travailler » sur la cascade de conséquences physiologiques et métaboliques à la suite d'une blessure à la tête, et non pas uniquement à la gestion des activités du quotidien, activités trop nombreuses en l'état.

Ainsi, la règle d'or « faire peu, se reposer beaucoup » a largement été discutée avec FB. Et, le repos peut difficilement être abordé sans aborder en parallèle la notion de fatigue. Dès lors, une bonne partie des séances est dédiée à la discussion de la fatigue. Il est important d'expliquer au sportif que la fatigue est très fréquente après une $C C$ dans le sport (Gosselin, 2009), mais également de manière plus générale à la suite d'un TCL, et ce même à long terme (Ma et al., 2019). Cette fatigue impacte de nombreux paramètres de la vie quotidienne (Fortier-Lebel \& Dupont, 2019). Avec l'expérience clinique et afin de faciliter la compréhension du processus, j'ai imagé la résistance humaine avec l'idée que l'on possède trois piles. Cela s'explique de la manière suivante : chaque personne possède trois piles d'énergie, une pile qui fonctionne à court terme $(\mathrm{CT})$, une pile qui fonctionne à moyen terme (MT) et une pile qui fonctionne à long terme (LT).

La pile à $\boldsymbol{C T}$. Elle représente l'énergie de la journée. Techniquement, si la nuit de sommeil a été bonne, elle peut sans autre permettre de passer une bonne journée. Avec une fatigue normale de fin de journée, elle aura la nuit pour se recharger et permettre de gérer la journée suivante. Si la nuit de sommeil n'est pas suffisante, on va puiser dans la pile à MT pour gérer sa journée. La pile à CT est celle qui varie le plus.

La pile à MT. Elle représente l'énergie de la semaine, qui va apporter une baisse de jour en jour, avec un vendredi qui est plus difficile et le besoin du week-end pour se recharger. Quand les semaines et week-ends ne permettent pas de se recharger suffisamment, on va puiser dans la pile à LT.

La pile à $\mathbf{L T}$. Elle représente l'énergie sur plusieurs mois et qui fait que les vacances sont souvent les bienvenues après quelques mois d'activités. C'est la pile qui varie le moins et celle qui doit être la plus remplie, afin d'assurer une base stable, dans laquelle puiser, quand cela est nécessaire.

À la suite d'une lésion/blessure cérébrale, ces trois piles sont vides, car il a fallu puiser dans toutes les réserves à disposition pour gérer les séquelles cérébrales et toute la cascade de modifications physiologiques, métaboliques et

\footnotetext{
${ }_{3}^{2}$ Naturellement, 11 est précisé que le processus est bien plus complexe.

${ }^{3} \mathrm{Au}$ plus tard la finir à $16 \mathrm{~h}$; maximum $1 \mathrm{~h}$; seulement si le sommeil nocturne est bon; pas dans son lit; mettre un réveil.

${ }^{4}$ Cohérence cardiaque : respiration régulière pour permettre un contrôle du rythme cardiaque.

${ }^{5}$ Se laisser aller aux pensées qui viennent et qui partent. A faire seulement si cela ne crée aucune anxiété.
}

${ }^{6}$ Le fait que le film ou le livre soit déjà connu permet de réduire la charge de la concentration pour suivre l'histoire.

cognitives qui en découlent ${ }^{2}$. Comme elles sont vides, il n'y a quasi aucune marge de manœuvre et la personne ne vit que sur les réserves de la pile à $\mathrm{CT}$, sous condition naturellement que la nuit de sommeil soit réparatrice. Comme cela est souvent l'inverse au début de la blessure, cela explique pourquoi les personnes ressentent une fatigue très importante, fréquente, et pour toutes activités de la vie quotidienne. Afin de rééquilibrer tout cela, la notion du repos devient alors capitale, pour permettre de remplir à nouveau les trois piles, mais surtout celle à LT qui représente la base de stabilité. Comme mentionné plus haut, il ne suffit pas juste de dire « repose-toi ». Il faut détailler, expliquer, donner des exemples, voire même faire une planification stricte des activités et du repos (Beni, 2019). Naturellement, la qualité du sommeil est investiguée et le cas échéant traitée.

Les activités au quotidien. Si la fatigue est la plainte unique, une prise en charge ciblée sur cet aspect peut être conduite (Beni, 2010). Mais dans le cadre des CC, la fatigue n'est pas l'unique problème et d'autres symptômes sont présents tel qu'on peut l'observer à travers les plaintes rapportées par FB (Annexe A). La problématique est alors élargie de manière à tenir compte de toute la symptomatologie. De ce fait, il faut travailler sur les activités du quotidien que FB peut réaliser, en tenant compte du seuil des symptômes. Pour ce faire, FB a rempli un tableau (Annexe B), composé de trois colonnes. En résumé, il est demandé à la personne de réfléchir aux activités de la journée qui font augmenter les symptômes et à celles qui font diminuer ces symptômes. Puis, en tenant compte de la description des piles ci-dessus, la personne pourra choisir son activité en fonction du coût qu'elle engendre. La colonne activités qui diminue les symptômes est souvent difficile à remplir. La sieste ne pouvant pas être l'unique source de repos, il faut trouver comment s'occuper sans que cela vide sa pile. Chaque personne doit trouver ce qui la repose et/ou la ressource. Les sportifs manquent souvent d'idées à ce sujet, car le repos n'est pas tellement dans leurs habitudes de vie. Ainsi, des idées d'activités qui reposent/ressourcent/diminuent les symptômes ont été données à $\mathrm{FB}$ dont la sieste ${ }^{3}$, la relaxation, la respiration, la cohérence cardiaque ${ }^{4}$, la méditation, la sophrologie, un bain chaud ou une douche, écouter de la musique douce, boire une tisane calmante, s'occuper d'un animal, dessiner, vagabondage de pensées ${ }^{5}$, regarder des photos (si possible, pas devant un écran) et se rappeler les bons souvenirs associés. Dans les activités neutres, regarder un film connu, lire un livre déjà $1 \mathrm{u}^{6}$, lire une bande dessinée, faire du jardinage ainsi que cuisiner peuvent être proposées. Chaque individu étant différent dans l'expression de sa symptomatologie, la personne devra par elle-même créer sa propre liste, aussi sur la base de ce qu'elle aime faire.

Une fois ces trois colonnes remplies, afin de permettre le bon choix d'activités, deux autres images, à choix, ont été présentées à $\mathrm{FB}$ :

- Le compte en banque : En début de journée, un certain montant d'argent est présent dans le compte et certaines activités vont le vider et d'autres vont le remplir ou le garder intact. Les activités qui augmentent les symptômes vident le compte et celles qui font diminuer les symptômes 
le remplissent. Ainsi, si le compte est vide, il faut choisir les activités qui font baisser les symptômes, afin de renflouer le compte et donc permettre de faire à nouveau une activité plus demandante. (Powell, 2015).

- Le régime alimentaire par système de points : Chaque jour, nous disposons d'une quantité de points qui nous guide dans ce que l'on peut ou non manger. Chaque aliment est doté d'un nombre de points, établi sur la base calorique de celui-ci. Chaque fois qu'un aliment est mangé, le nombre de points à disposition diminue. De la même manière, l'activité choisie doit être décidée en fonction des points qui restent à disposition pour la fin de la journée (activités qui augmentent les symptômes). Dans la perte de poids, des points peuvent être gagnés par l'activité physique, ici cela sera par les activités qui diminuent les symptômes. Cette idée de faire un parallèle avec un régime alimentaire est issue de ma pratique clinique.

Ce système de gestion des symptômes et de choix d'activités a été couplé au concept clé «maximiser ce qui fait du bien et minimiser ce qui fait du mal » (Beni, 2019). La marche extérieure a été imposée à FB, comme activité quotidienne, car les bienfaits d'une activité à faible intensité sur le processus de récupération sont aujourd'hui largement reconnus (Bailey et al., 2019). La marche est donc imposée à tout sportif qui consulte à la suite d'une CC.

L'accompagnement dans l'arrêt de la pratique du hockey. Compte tenu des plaintes de FB, des séquelles persistantes à long terme et du nombre de $\mathrm{CC}$, il était important d'aborder l'arrêt de la pratique du hockey. Ainsi, toujours sous le principe de transparence, les risques à court, moyen et long terme des $\mathrm{CC}$ ont été expliqués en détail à FB. Naturellement, FB était très réfractaire à l'idée de devoir stopper sa carrière, mais surtout d'arrêter de pratiquer son sport-passion. Il a fallu travailler sur différents points. Ils sont très résumés ci-dessous, mais ils ont été discutés et repris sur plusieurs séances.

-Tout d'abord, il a longuement été discuté de son identité de hockeyeur. Il a commencé le hockey à l'âge de 5 ans : c'était son identité. Cela l'a construit, modelé et occupé durant toutes ces années. Il a fallu assouplir cette identité, en la transposant de hockeyeur, à celle du sportif en général. L'idée étant qu'il soit prêt à s'ouvrir à d'autres sports. Des techniques issues des thérapies cognitivocomportementales sur l'acceptation du changement ont été appliquées, comme la balance décisionnelle ou la flèche descendante, c'est-à-dire permettre à la personne «de se décentrer par rapport au point de vue initial et de remplacer progressivement ses pensées automatiques par des pensées alternatives» (Mihaescu et al.,1998 p.252; Ovide \& Fontaine, 2011).

- Ensuite, une analyse du pourquoi il aimait autant pratiquer le hockey sur glace a été conduite. La notion d'adrénaline que procure la pratique du hockey sur glace, comme dans de nombreux sports de contact, a été rapidement identifiée. Il recherche et aime cette sensation. Mais comme les sports de contact au sens large lui sont interdits, il devenait évident que l'adrénaline n'allait pas devoir se ressentir dans le contact, mais dans d'autres actions du nouveau sport choisi. À nouveau, en appliquant des stratégies issues des thérapies cognitivocomportementales, les sports alternatifs ont été dégagés, en mettant en évidence leur force, comme la stratégie du jeu ou l'endurance.

-Un détour par la notion de l'autodestruction a aussi été fait. Il lui a été confirmé que les troubles pourraient encore s'aggraver et s'intensifier s'il persistait à jouer au hockey, et cela sans même avoir de CC (c.-à-d., notion de souscommotion) et que l'amendement de ses troubles n'était pas certain du tout, même après l'arrêt.

-Il a fallu aussi aborder les aspects de la satisfaction des besoins dans la pratique du sport. D'une manière générale, la notion de satisfaction est très souvent réduite dans le cas de TCL, provoquant des états anxio-dépressifs (AuclairPilote et al., 2019). Pour toutes ces raisons, il était important que $\mathrm{FB}$ puisse s'adonner à une autre activité sportive, afin de maintenir un niveau de satisfaction personnelle et d'équilibre de vie.

-FB a eu aussi la possibilité de discuter avec d'anciens amis hockeyeurs, qui avaient dû eux aussi mettre un terme à leur carrière à cause d'une ou plusieurs $\mathrm{CC}$. Avoir pu discuter avec des pairs qui puissent comprendre ce qu'il ressentait a été un point capital dans la prise de décision. D'ailleurs, dans l'acceptation du changement, il faudrait toujours conseiller à la personne de pouvoir discuter avec des personnes qui ont le même vécu. Des personnes qui appartiennent au même groupe de référence permettent une meilleure résonnance. En effet, le fait d'appartenir à une collectivité permet de partager avec les autres membres des idées ou des traits communs, pour pouvoir se reconnaître dans le « nous » (Rocher, 1972).

\section{L'évaluation neuropsychologique}

Le bilan a été réalisé 11 mois après la $1^{\text {re }}$ consultation. Il a été effectué en deux séances : la première séance a permis de récolter les données d'anamnèse et les plaintes actuelles. La seconde séance a été dédiée à l'administration des tests.

Tests. Les tests classiques ont été choisis pour une personne jeune et surtout pour évaluer les domaines classiquement altérés après un TCL, à savoir les capacités mnésiques, attentionnelles et exécutives.

L'annexe $\mathrm{C}$ répertorie tous les tests administrés, ainsi que les résultats et l'interprétation.

\section{Plaintes cognitives, émotionnelles et comportementales (J+11 mois)}

Spontanément sur un plan cognitif, FB se plait de difficultés de lecture, en lien avec des difficultés de saccades. Il a des pertes de mots dans les langues qu'il pratique couramment, en se trompant par exemple sur la conjugaison. Il oublie aussi les prénoms des personnes. Sur un plan physique, quand il fait certains mouvements brusques, il releve des vertiges. Il a de légers maux de tête quand il doit lire de manière continue et intense.

Sur demande spécifique, l'orientation, le raisonnement, l'écriture et la planification sont en ordre. Sur le plan émotionnel, il va globalement bien. Sur le plan du comportement, il a encore occasionnellement des sautes d'humeur, surtout quand il est fatigué. Dans la réalisation 
de son travail, il relève une plus grande fatigabilité, une irritabilité plus marquée et une augmentation des sautes d'humeur, avec la nécessité de s'isoler de ses collègues.

Apparition et évolution. Tous ses troubles sont apparus surtout depuis le TCC au Jour J - 10 ans. Les troubles ont persisté longtemps, sans jamais vraiment disparaître, avec de nouveaux symptômes à la suite des TCL suivants. Les troubles se sont possiblement amendés entre J-4 ans et J-2 ans, mais il a développé une forte dépression qui a probablement masqué les symptômes, étant donné qu'il ne réalisait aucune activité. Ensuite, les troubles ont repris quand il a recommencé à jouer au hockey (c.-à-d., au J-3 mois) et ils sont fortement réapparus à la suite du TCL qui a déclenché la consultation du Jour J.

Comportement. Au niveau du comportement FB est collaborant, adéquat et il ne se laisse pas distraire par des éléments extérieurs. Au niveau de la passation des tests, FB est rapide et efficace. Lors de tâches plus demandantes, il ressent une pression dans le crâne, des difficultés à réaliser des tâches visuelles ou des tâches longues. Une fatigue a été constatée après environ une heure et demie d'évaluation, qui a également provoqué une hypersensibilité à la lumière.

\section{Résultats}

\section{Résultats de la prise en charge}

Deux axes de prise en charge avaient été définis : 1) la gestion des symptômes post-commotionnels et 2) l'accompagnement dans l'arrêt de la pratique du hockey.

En ce qui concerne la gestion des symptômes, lors des deux premières séances, les éléments suivants ont été abordés : l'anamnèse, les plaintes, les règles d'or, le repos et la fatigue ainsi que les questions de FB. Les séances suivantes ont permis de reprendre ces propos et d'aborder l'accompagnement dans l'arrêt de la pratique du hockey. Les deux premières séances ont été réalisées avec un délai d'une semaine et les suivantes avec un délai de deux semaines. Dans le cas de FB, il était important de rapprocher les premières séances, afin de lui fournir les différentes règles d'or rapidement, mais ensuite cela a été espacé pour qu'il puisse mettre en application les stratégies discutées et réfléchir aux propos (c.-à-d., l'application des concepts de l'adhérence au traitement et de la psychoéducation). Toutefois, selon les cas, il arrive qu'il faille faire plusieurs séances hebdomadaires de suite, avant de commencer à espacer les séances.

Grâce au seuil des symptômes qui monitore le choix des activités et leur durée, les jours qui ont suivi la première consultation ont permis à FB de s'auto-observer et de s'autoréguler, sur la base des outils expliqués ci-dessus. L'autorégulation est très importante, car elle offre au sportif une certaine maîtrise de la situation. FB a pu se rendre compte qu'il n'est pas toujours évident de faire le bon choix d'activités. D'ailleurs, les premiers jours FB était fréquemment au-dessus du seuil des symptômes. Mais, de jour en jour, il a appris à se monitorer et à appliquer des choix adéquats d'activités, en fonction du seuil des symptômes, et en appliquant en parallèle les bons temps de repos. Lors de la $3^{\mathrm{e}}$ séance, il est apparu que l'application adéquate des stratégies commençait à apporter des bénéfices et une réduction des symptômes. Il devenait alors capital d'aborder avec FB la notion des risques dans la poursuite de la pratique du hockey sur glace et surtout d'envisager d'arrêter ce sport.

Ainsi, sur cet aspect d'arrêter la pratique du hockey, la décision finale a été prise à la suite de nos discussions et des éléments fournis qui lui ont permis d'élaborer sa réflexion en dehors de nos séances, notamment en discutant avec ses pairs. L'arrêt a aussi été possible, car FB présentait des symptômes post-traumatiques assez marqués et il devenait évident que cela n'était plus possible pour lui de continuer une activité qui était en train de le détruire. Tout cela lui a permis de s'ouvrir à d'autres activités sportives, afin de lui permettre de s'adonner à des activités sportives satisfaisantes, comme il l'a fait toute sa vie. Il a d'ailleurs commencé à jouer au tennis, où la satisfaction dans l'endurance et les stratégies de jeu ont pris le dessus sur l'adrénaline du hockey sur glace. Cet accompagnement a également permis un arrêt de son sport-passion de manière sereine, sans développer un état anxiodépressif, comme cela avait été le cas entre le $\mathrm{J}-4$ ans et le $\mathrm{J}-2$ ans.

En résumé et comme cela est montré à l'Annexe $\mathrm{A}$, il y a une différence considérable dans la présence des symptômes et de leur intensité. En cela, la prise en charge a été bénéfique, car elle lui a appris à mieux comprendre ses symptômes, comment ils se déclenchent et comment les faire diminuer. Le fait d'avoir arrêté la pratique d'un sport qui continuait à alimenter les troubles a aussi apporté un bénéfice substantiel à la diminution des symptômes.

\section{Résultats du bilan neuropsychologique}

Sur la base des tests administrés (Annexe C), le bilan neuropsychologique effectué chez FB a mis en évidence un déficit dans une tâche de lecture, où le résumé est erroné, car il n'a pas compris ce qu'il a lu, associé à des performances possiblement déficitaires en apprentissage. Ensuite, les tâches qui demandent un fort engagement visuel sont déficitaires (c.-à-d., copie de la figure complexe de Rey, une tâche qui mesure la capacité à déplacer son focus attentionnel et lecture et abandon d'une tâche d'attention soutenue).

Le reste du tableau cognitif se situe dans les normes, voire dans les normes supérieures, compte tenu de son âge, sexe et nombre d'années d'étude. Sur un plan clinique, FB relève une pression sur la tête, plus marquée au niveau des tempes, lors de tâches demandantes. Cette pression s'est atténuée dès que la tâche s'est terminée. La réalisation d'une tâche demandant de focaliser une cible présente sur un écran d'ordinateur a été très difficile et a été même interrompue. En effet, il ne distinguait plus les formes, son regard n'arrivait pas à rester focalisé sur l'écran et il avait mal à la tête. Enfin, la lecture a été problématique, car cela lui a demandé une forte concentration oculaire (en lien avec ses problèmes de saccades) et il s'est montré alors incapable de comprendre ce qu'il lisait. Une fatigabilité était présente en fin de séance, ce qui a eu un impact sur ses performances. Il est devenu aussi sensible à la lumière. Il a constaté également une irritabilité plus marquée avec la fatigue. 
Tous ces troubles ont un impact dans le quotidien de $\mathrm{FB}$, que ce soit dans sa capacité à apprendre de nouvelles choses, à lire un document ou un livre, ou même à réaliser certaines tâches qui demandent de la focalisation. En raison de cela, FB s'est vu obligé de mettre en place des stratégies palliatives (p. ex., noter, faire des résumés, répéter plusieurs fois l'information). Sur le plan comportemental, FB a dû également veiller à se contrôler et/ou à éviter certaines situations sociales.

\section{Discussion}

L'objectif de ce chapitre était multiple. Après avoir très brièvement présenté les connaissances médicales théoriques actuelles des séquelles post-commotionnelles, une étude de cas d'un défenseur de hockey sur glace a été proposée. Cette étude de cas a permis d'offrir un éclairage important sur les séquelles cognitives, physiques et psychopathologiques à long terme de multiples $\mathrm{CC}$, en présentant un bilan neuropsychologique complet, à un an de la dernière $\mathrm{CC}$, et une prise en charge ciblée des séquelles post-commotionnelles de ce hockeyeur.

Grâce aux outils, aux stratégies et aux méthodes employées dans la prise en charge, les troubles de FB ont pu diminuer (Annexe A) et il a obtenu une aide efficace dans la décision d'arrêter le hockey sur glace. Ces éléments de prise en charge, axés sur les difficultés de FB, compilent une approche neuropsychologique et une approche issue des thérapies cognitivo-comportementales, toutes deux des approches complémentaires pour ce type de problématique. Les outils, les stratégies et les méthodes proposées dans cet article offrent la possibilité de réduire les SPC et ils pourront être des aides précieuses pour toute personne qui aura en charge des sportifs commotionnés. En effet, la littérature est maigre sur les aides pratico-pratiques, car très souvent les conseils fournis sont très généraux. Ces outils, ces stratégies et ces méthodes n'ont, a priori, jamais été publiés. En outre, l'histoire de FB n'est pas unique et elle met en lumière les lacunes du milieu médical trop souvent démuni et peu compétent dans la gestion des $\mathrm{CC}$ à répétition, tant sur les règles d'arrêt de la pratique de l'activité à risque, que sur l'explication des symptômes post -commotionnels. Les neuropsychologues ont ici un rôle important à jouer et ils doivent naturellement être compétents dans le domaine de la neuropsychologie (Van der Linden, 2018), mais plus spécifiquement dans la neuropsychologie du sport (McCrory et al., 2017).

Ensuite, un bilan neuropsychologique complet a été réalisé à une année de la dernière CC. Les différents troubles présentés par $\mathrm{FB}$ à ce bilan sont compatibles avec les séquelles des multiples CC survenues dans la pratique du hockey sur glace; ces troubles ne s'expliquent pas par d'autres facteurs et ils sont qualifiés de persistants (McCrory et al., 2017). Les séquelles principales sont probablement liées au premier TCC (c.-à-d., au Jour J - 10 ans), où des lésions cérébrales, montrées à la neuroimagerie, persistent toujours aujourd'hui. Compte tenu du délai depuis la dernière $\mathrm{CC}$, il est probable que les symptômes décrits persistent à vie, comme cela a été montré par de nombreux auteurs (Tator et al., 2016). De plus, FB présente plusieurs facteurs aggravants à la persistance des symptômes à long terme (c.-à-d., un nombre important de $\mathrm{CC}$; des $\mathrm{CC}$ qui n'ont jamais été bien soignées ; une durée de récupération très longue, voir même il n'a jamais vraiment récupéré puisqu'il présente encore des symptômes post-commotionnels ; des petits chocs ont provoqué des $\mathrm{CC}$; les premières $\mathrm{CC}$ et les premiers chocs ont eu lieu alors que son cerveau n'était pas encore mature ; et la présence d'un court délai entre certaines $\mathrm{CC}$ ).

Cet article présente des limites. Tout d'abord, il manque les données médicales des premières $\mathrm{CC}$ de $\mathrm{FB}$, notamment les données de l'IRM cérébrale. Cela aurait permis une comparaison avec l'IRM réalisée à $\mathrm{J}+11$ mois. Ensuite, les éléments de prise en charge présentés dans cet article sont spécifiques à la problématique de $\mathrm{FB}$ et donc limités. En effet, une prise en charge qui se veut individualisée sera différente pour un autre individu et d'autres éléments de prise en charge devront être envisagés, le cas échéant. Enfin, le bilan neuropsychologique a été effectué à 11 mois de la dernière $\mathrm{CC}$. Un bilan neuropsychologique de contrôle d'ici deux à trois ans permettrait de confirmer ou non la persistance des symptômes à vie. Mais l'intérêt serait aussi de voir comment les troubles de FB vont évoluer d'ici 15 à 20 ans, et notamment d'observer si FB développe ou non une encéphalopathie chronique traumatique (McKee et al., 2016).

\section{Conclusion}

L'avenir du diagnostic des $\mathrm{CC}$ réside dans les progrès de la neuroimagerie ou des marqueurs sanguins (Shenton et al., 2018). Mais une prise en charge de qualité restera toujours nécessaire. Il n'y a pas de consensus national ou international quant au nombre de $\mathrm{CC}$ rédhibitoire pour arrêter une activité sportive à risque. Toutefois, le cumul de tous les facteurs aggravants décrits ci-dessus est suffisant pour être catégorique dans la nécessité d'arrêter la pratique de ladite activité à risque. Aucun des médecins que $\mathrm{FB}$ a consulté durant toutes ces années n'avaient pris le temps de discuter avec lui de la nécessité de se soigner adéquatement, afin d'éviter des séquelles à long terme. Cela rejoint le constat que certains médecins sont encore aujourd'hui démunis dans la gestion des CC (Stoller et al., 2014). Dès lors, face à des séquelles persistantes, comme pour FB ou dans 23\% des cas (Tator et al., 2016), un accompagnement professionnel, individualisé et transparent doit pouvoir être offert au sportif. Le but étant naturellement d'agir le plus adéquatement et précocement possible sur les séquelles, afin de raccourcir le temps d'arrêt de jeu et d'éviter les séquelles à long terme (Castile et al., 2012). Dès lors que des séquelles deviennent persistantes et fortement handicapantes, l'arrêt de la carrière doit être envisagé et discuté. Face à ce changement d'identité, un accompagnement qualifié et professionnel doit être offert, afin d'éviter le développement d'états anxio -dépressifs et/ou de mauvais diagnostics psychiatriques. Les techniques issues des thérapies cognitivocomportementales offrent une approche efficace.

$\mathrm{Au}$ final, les CC dans le sport sont toujours plus fréquentes. Premièrement, car la prise de conscience de cette problématique de santé est meilleure, mais aussi, car le nombre de $\mathrm{CC}$ augmente pour différentes raisons (p. ex., matériel, règles, gabarit du sportif). Le risque est présent et il est du devoir des professionnels de la santé de veiller à bien soigner les sportifs victimes d'une CC. Toutefois, certaines fédérations sportives restent encore très frileuses 
face à cette réalité médicale, notamment en raison d'énormes enjeux financiers, mais aussi sportifs. FB n'a pas été informé des séquelles possibles à long terme et cela pose de nombreuses questions éthiques à vouloir encore aujourd'hui banaliser ou minimiser l'impact des CC à répétition (Gillett, 2018). En effet, même si aujourd'hui la survenue de l'encéphalopathie chronique traumatique reste controversée, il paraît clair qu'à la suite de $\mathrm{CC}$ à répétition, notamment si elles sont mal soignées, des séquelles persistantes sont certaines. Le cas de FB en témoigne. Ces séquelles handicapent le quotidien, tant sur un plan personnel que professionnel, chez des personnes qui très souvent n'ont pas encore eu trente ans.

\section{« Si tu n'arrives pas à penser, marche}

\section{Si tu penses trop, marche}

Si tu penses mal, marche encore » Jean Giono

\section{Références}

Alsalaheen, B., Stockdale, K., Pechumer, D., \& Broglio, S. P. (2016). Validity of the Immediate Post Concussion Assessment and Cognitive Testing (ImPACT). Sports Medicine, 46, 1487-1501.

Auclair-Pilote, J., Lalande, D., Tinawi, S., Feyz, M., \& de Guise, E. (2019). Satisfaction of basic psychological needs following a mild traumatic brain injury and relationships with post-concussion symptoms, anxiety, and depression. Disability and Rehabilitation, 43, 1-9.

Bailey, C., Meyer, J., Briskin, S., Tangen, C., Hoffer, S. A., Dundr, J., Brennan, B., \& Smith, P. (2019). Multidisciplinary Concussion Management: A Model for Outpatient Concussion Management in the Acute and Post-Acute Settings. The Journal of Head Trauma Rehabilitation, 34(6), 375-384.

Beni, C. (2019). Les Commotions Cérébrales dans le sport. In Roberta Antonini Philippe (Ed.), 10 cas pratiques en psychologie du sport (pp. 51-74). Dunod.

Beni, C. (2010). Prise en charge de la fatigue suite à un traumatisme crânien : Application des stratégies cognitivo-comportementales. In B. Zellner Keller (Ed.), Des métiers pour aider : Apport de l'approche cognitivo-comportementale et de ses outils. Médecine et Hygiène.

Beni, C., Rochat, L., Malysse, N., Delecroix, H., Arnould, A., Azouvi, P., ... Van der Linden, M. (2017). L'échelle des changements de comportements socio-émotionnels de Genève (ECCSEG) : Validation auprès de patients victimes d'un traumatisme crânio-cérébral. Canadian Journal of Behavioural Science / Revue Canadienne Des Sciences Du Comportement, 49, 7-17.

Brooks, B. L., Mannix, R., Maxwell, B., Zafonte, R., Berkner, P. D., \& Iverson, G. L. (2016). Multiple Past Concussions in High School Football Players: Are There Differences in Cognitive Functioning and Symptom Reporting? The American Journal of Sports Medicine, 44, 3243-3251.
Castile, L., Collins, C. L., McIlvain, N. M., \& Comstock, R. D. (2012). The epidemiology of new versus recurrent sports concussions among high school athletes, 20052010. British Journal of Sports Medicine, 46, 603.

Ellis, M. J., Leiter, J., Hall, T., McDonald, P. J., Sawyer, S., Silver, N., Bunge, M., \& Essig, M. (2015). Neuroimaging findings in pediatric sports-related concussion. Journal of Neurosurgery: Pediatrics, 16, $241-247$.

Fontaine, O. \& Fontaine P. (2011). Guide clinique de thérapie comportementale et cognitive. RETZ.

Fortier-Lebel, O. \& Dupont, C. (2019). Revue narrative de l'effet des traumatismes crâniens sur la fatigue. Neuropsychologie Clinique et Appliquée / Applied and Clinical Neuropsychology, 3, 82-91.

Frémont, P. (2017, December). [Lectures notes]. Commotion cérébrale : prévention, détection et gestion dans mon milieu, Université de Laval.

Gillett, G. (2018). Concussion in Sport: The Unheeded Evidence. Cambridge Quarterly of Healthcare Ethics, 27, 710-716.

Gosselin, N., Lassonde, M., Petit, D., Leclerc, S., Mongrain, V., Collie, A., \& Montplaisir, J. (2009). Sleep following sport-related concussions. Sleep Medicine, 10, 35-46.

Harmon, K. G., Drezner, J. A., Gammons, M., Guskiewicz, K. M., Halstead, M., Herring, S. A., ... Roberts, W. O. (2013). American Medical Society for Sports Medicine position statement: concussion in sport. British journal of sports medicine, 47, 15-26.

Howell, D. R., Taylor, J. A., Tan, C. O., Orr, R., \& Meehan, W. P. (2018). The Role of Aerobic Exercise in Reducing Persistent Sport-related Concussion Symptoms. Medicine and Science in Sports and Exercise, 51, 647-652.

Ma, H. P., Chen, P. S., Wong, C. S., Chang, C. F., Ou, J. C., Tsai, Y. R., ... Wu, J. C. C. (2019). Psychometric Evaluation of Anxiety, Depression, and Sleep Quality after a Mild Traumatic Brain Injury: A Longitudinal Study. Behavioural Neurology, 2019, 1-9.

Manley, G., Gardner, A. J., Schneider, K. J., Guskiewicz, K. M., Bailes, J., Cantu, R. C., ... Iverson, G. L. (2017). A systematic review of potential long-term effects of sport-related concussion. British Journal of Sports Medicine, 51, 969-977.

Mansell, J. L., Tierney, R. T., Higgins, M., McDevitt, J., Toone, N., \& Glutting, J. (2010). Concussive signs and symptoms following head impacts in collegiate athletes. Brain Injury, 24, 1070-1074. j.nec.2016.05.009

McCrory, P., Meeuwisse, W., Dvorak, J., Aubry, M., Bailes, J., Broglio, S., ...Vos, P. E. (2017, October). Consensus statement on concussion in sport [conference session] International conference on concussion in sport, Berlin.

McCrory, P., Davis, G., \& Makdissi, M. (2012). Second impact syndrome or cerebral swelling after sporting head injury. Current Sports Medicine Reports, 11, 2123.

McKee, A. C., Alosco, M. L., \& Huber, B. R. (2016). Repetitive Head Impacts and Chronic Traumatic Encephalopathy. Neurosurgery Clinics of North America, 27, 529-535. 
Mez, J., Daneshvar, D. H., Kiernan, P. T., Abdolmohammadi, B., Alvarez, V. E., Huber, B. R., ... McKee, A. C. (2017). Clinicopathological Evaluation of Chronic Traumatic Encephalopathy in Players of American Football. JAMA, 318, 360-370.

Mihaescu, G., Séchaud, M. C., Delsignore, A., \& Cottraux, J. (1998) Précis de thérapie Comportementale et Cognitive. Médecine et Hygiène.

Mittenberg, W., Tremont, G., Zielinski, R. E., Fichera, S., \& Rayls, K. R. (1996). Cognitive-behavioral prevention of postconcussion syndrome. Archives of Clinical Neuropsychology, 11, 139-145.

Organisation mondiale de la santé (Éd.). (2009). Classification statistique internationale des maladies et des problèmes de santé connexes: CIM-10 (10e révision). Organisation mondiale de la santé.

Powell, T. (2015). Exercice de remédiation cognitive pour les adultes cérébrolésés (Vol. 1). De Boeck Supérieur.

Purcell, L. K. \& Canadian Paediatric Society, Healthy Active Living and Sports Medicine Committee. (2014). Sport-related concussion: Evaluation and management. Paediatrics \& Child Health, 19, 153-165.

Rocher, G (1972). Introduction à la sociologie générale. Hurtubise.

Shenton, M. E., Price, B. H., Levin, L., \& Edersheim, J. G. (2018). Mild traumatic brain injury: Is DTI ready for the courtroom? International Journal of Law and Psychiatry, 61, 50-63.

Stoller, J., Carson, J. D., Garel, A., Libfeld, P., Snow, C. L., Law, M., \& Frémont, P. (2014). Do family physicians, emergency department physicians, and pediatricians give consistent sport-related concussion management advice? Canadian Family Physician, 60, 550-552.

Tator, C. H., Davis, H. S., Dufort, P. A., Tartaglia, M. C., Davis, K. D., Ebraheem, A., \& Hiploylee, C. (2016). Postconcussion syndrome: demographics and predictors in 221 patients. Journal of Neurosurgery, 12, 12061216.

Van der Linden, M. (2018). Pour une neuropsychologie clinique intégrative et centrée sur la vie quotidienne. Revue de neuropsychologie, 10, 41-46.

Reçu le 16 février 2020

Révisé le 25 avril 2020

Accepté le 16 mai 2021 
Annexe A

Liste des symptômes post-commotionnels de FB lors du Jour J et Jour J + 11

\begin{tabular}{|c|c|c|c|c|c|c|}
\hline \multirow[b]{2}{*}{ Symptômes Physiques } & \multicolumn{3}{|c|}{ Jour J } & \multicolumn{3}{|c|}{ Jour $J+11$ mois } \\
\hline & Oui & Non & $\begin{array}{c}\text { Si oui, } \\
\text { intensité } / 10 *\end{array}$ & Oui & Non & Si oui, intensité / 10 * \\
\hline Mal de tête & & & 9 & & & \\
\hline Pression dans le crâne & & & 8 & & & \\
\hline Vertiges & & & 8 & & & \\
\hline HS bru it & & & 6 & & & \\
\hline HS lumière & & & 8 & & & 5 \\
\hline Vison trouble & & & 8 & & & 1 \\
\hline Sensation d'être dans le brou illard & & & 5 & & & 2 \\
\hline Sensation d'an omalie & & & 5 & & & \\
\hline Sens ation d'être ralenti phy siquement & & & 3 & & & \\
\hline \multicolumn{7}{|l|}{ Autre : } \\
\hline Symptômes Cognitifs & Oui & Non & $\begin{array}{c}\mathrm{Si} \text { oui, } \\
\text { intensité } / 10 *\end{array}$ & Oui & Non & Si oui, intensité / 10 * \\
\hline Oublis & & & 8 & & & \\
\hline Difficultés d'apprentis sage & & & 10 & & & 2 \\
\hline Problèmes de concentration & & & 10 & & & 5 \\
\hline Difficultés à prendre des décisions & & & & & & 2 \\
\hline Ralentissement dans les idées & & & 3 & & & 2 \\
\hline Autre : Lecture & & & 10 & & & 5 \\
\hline Sommeil et fatigue & Oui & Non & $\begin{array}{c}\text { Si oui, } \\
\text { fréquence par } \\
\text { semaine }\end{array}$ & Oui & Non & $\begin{array}{c}\text { Si oui, fréquence par } \\
\text { semaine }\end{array}$ \\
\hline Fatigue & & & 7 & & & \\
\hline \multicolumn{7}{|l|}{ Somnolence } \\
\hline \multicolumn{7}{|l|}{ Hypers omnie } \\
\hline Insomnie & & & 5 & & & \\
\hline Difficultés d'en dormissement & & & 6 & & & \\
\hline Réveils noctumes & & & 7 & & & \\
\hline \multicolumn{7}{|l|}{ Cauchemars } \\
\hline \multicolumn{7}{|l|}{ Somn ifères } \\
\hline \multicolumn{7}{|l|}{ Autre: } \\
\hline Comportement et Emotion & Oui & Non & $\begin{array}{c}\text { Si oui, } \\
\text { intensité } / 10 *\end{array}$ & Oui & Non & Si oui, intensité / 10 * \\
\hline Emotivité accrue & & & 5 & & & \\
\hline Tristes se & & & 5 & & & \\
\hline Nervos ité & & & 10 & & & \\
\hline Irritabilité & & & 10 & & & \\
\hline Impatience & & & 8 & & & \\
\hline Agressivité & & & 3 & & & \\
\hline Autre: Sautes d'humeur & & & 10 & & & \\
\hline
\end{tabular}




\section{SÉQUELLES DE MULTIPLES COMMOTIONS CÉRÉBRALES}

\section{Annexe B}

Grille d'évaluation des activités du quotidien

\section{Grille d'évalution des activités du quotidien}

Etabli par Beni Catia, Docteur en Psychologie, Spécialiste en Neuropsychologie du Sport; mai 2020

Nom: $T B$ Date: Jow

Le tableau ci-dessous est composé de trois colonnes. Une colonne qui répertorie les activités du quotidien qui augmentent les symptômes post-commotionnels, une colonne qui répertorie les activités du quotidien qui diminuent les symptômes post-commotionnels, et une colonne qui répertorie les activités « neutres " du quotidien.

Merci de réfléchir et d'analyser les différentes activités de votre quotidien et d'évaluer dans quelle colonne elles doivent être placées. Une fois la colonne choisie, il vous faut encore évaluer l'intensité, sur une échelle comprise entre 1 et 10 ( 1 = impact très discret ; 10 = impact très fort).

Exemple :

- Si quand je lis un livre, cela me provoque des maux de tête, des nausées et des troubles de la concentration, je vais mettre cette activité dans la colonne " activités qui augmentent les symptômes " et évaluer l'intensité à $7 / 10$.

- A l'inverse, si faire une sieste fait disparaître tous mes symptômes, je vais mettre cette activité dans la colonne " activités qui diminuent les symptômes \#, et évaluer ce bénéfice à 10/10.

- Enfin, si cuisiner ne me provoque aucun symptôme, mais ne les fait pas non plus diminuer, alors je vais écrire cette activité dans la colonne " neutre ".

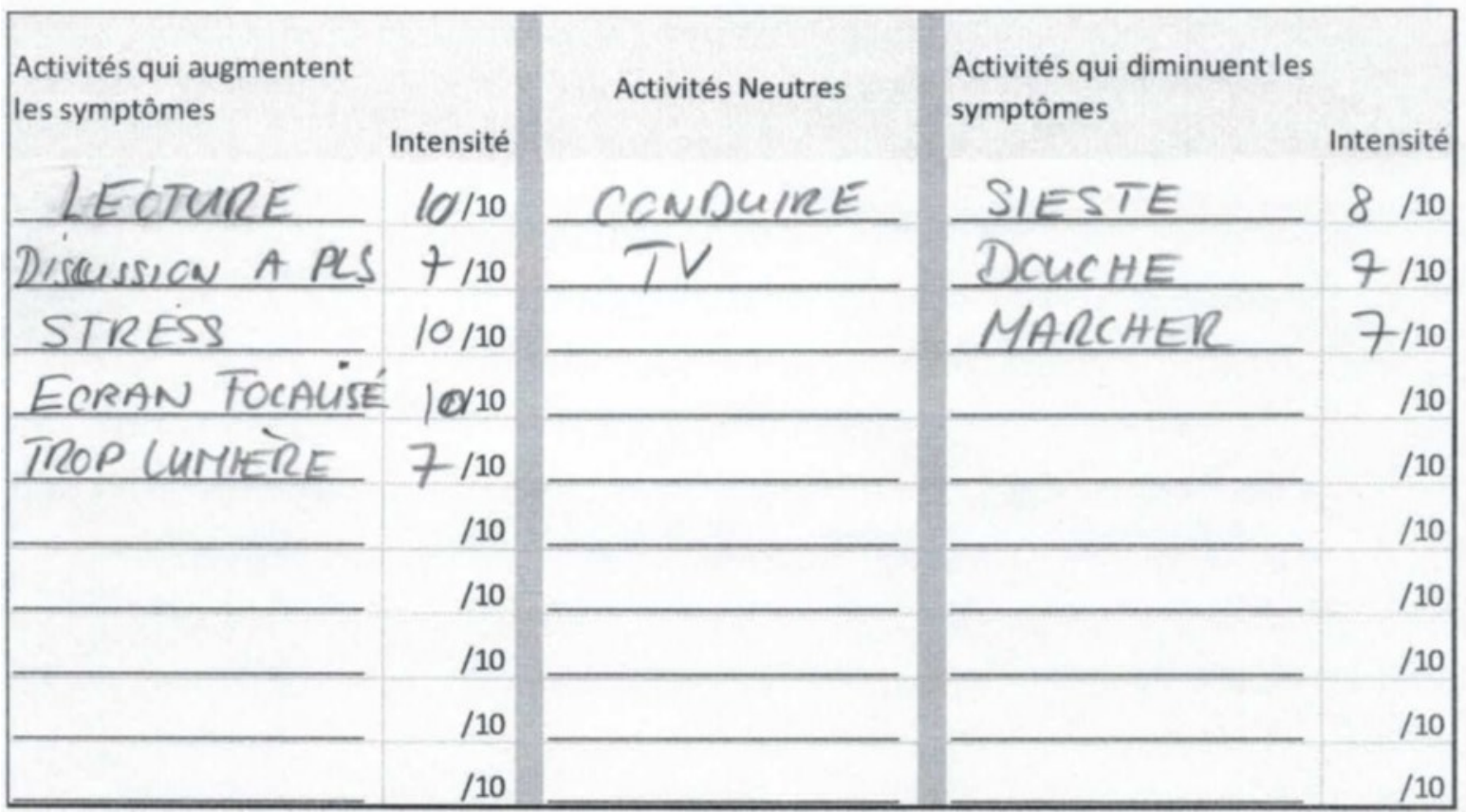

Note. Etabli par Beni Catia, Docteur en Psychologie, Spécialiste en Neuropsychologie du Sport. 
BENI

\section{Annexe C}

Tests, scores et interprétations des performances de FB à $J+11$ mois

\begin{tabular}{|c|c|c|c|c|}
\hline \multicolumn{2}{|c|}{ Domaines Cognitifs } & Tests et référence des tests & Score FB & Interprétation \\
\hline \multirow{6}{*}{ Langage } & Discours & Discours oral spontané & en ordre & Normes \\
\hline & $\begin{array}{l}\text { Dén omination } \\
\text { continue }\end{array}$ & $1^{\text {ère }}$ partie du Stroop Test (Delis \& Kaplan) & $27^{\prime \prime}$, NS10, 0 erreur & Normes \\
\hline & Lecture continue & 2ème partie du Stroop Test (Delis \& Kaplan) & $19^{\prime \prime}, \mathrm{NS} 12,0$ erreur & Normes \\
\hline & Lecture à hautev oix & Texte du Montréa1 - Tou louse & en ordre & Normes \\
\hline & Production écrite & Ecriture automatique (nom, prénom, adresse) & en ordre & Normes \\
\hline & Production écrite & Ecriture spontanée & en ordre & Normes \\
\hline Calcul & Calcul Oral & Sur la base du Test de la PASAT & en ordre & Normes \\
\hline Praxis & Praxies con structives & Copie de la Figure Complexe de Rey (1959) & $\begin{array}{c}30 / 36 ;<\operatorname{Pc} 5 \text { (approximative et réalisée } \\
\text { de manière scin dée) }\end{array}$ & Déficitaire \\
\hline \multirow[t]{2}{*}{ Orientation } & Orientation & Jour-Mois - A nnée - Semaine - Sais on & $5 / 5$ & Normes \\
\hline & Orientation spatiale & Lieu - Étage - Ville - Canton - Rég ion & $5 / 5$ & Normes \\
\hline \multirow{5}{*}{$\begin{array}{l}\text { Mémoire à } \\
\text { court terme } \\
\text { / de travail }\end{array}$} & Empan verbal direct & $\begin{array}{c}\text { Echelle d'intelligence WAIS-IV } \\
\text { (Wechsler, 2008) }\end{array}$ & empan de $6, \operatorname{Pc} 35$ & Normes \\
\hline & Emp an verbal indirect & $\begin{array}{l}\text { Echelle d'intelligence WAIS-IV } \\
\text { (Wechsler, 2008) }\end{array}$ & empan de 6, Pc84 & Normes \\
\hline & $\begin{array}{l}\text { Emp an verbal ord re } \\
\text { croissant }\end{array}$ & $\begin{array}{l}\text { Echelle d'intelligence WAIS-IV } \\
\text { (Wechsler, 2008) }\end{array}$ & empan $6, \operatorname{Pc} 53$ & Normes \\
\hline & Mémoire de travail & $\begin{array}{l}\text { Mémoire des Chiffres de la WAIS IV } \\
\text { (Wechsler, 2008) }\end{array}$ & $29 ;$ NS11 & Normes \\
\hline & $\begin{array}{l}\text { Mise à Jour de } \\
\text { l'information }\end{array}$ & $\begin{array}{c}\text { Test du PA SA T (Naegele et Mazza, } \\
\text { Adaptation fran çaise, 2004) }\end{array}$ & $59 / 60 ;$ Pc50 & Normes \\
\hline \multirow{5}{*}{$\begin{array}{l}\text { Mémoire } \\
\text { épisodique/ } \\
\text { apprentissage }\end{array}$} & Mémoire verbale & Rappel/Résumé texte lu (MT) & lacunaire, erroné & Déficitaire \\
\hline & & Buschke Selective Reminding Test (Buschke, & Moy d'apprentissage : 11,8 & Normes \\
\hline & & $\begin{array}{l}\text { 1973. RLS } 15 \text {-items, validé en français par } \\
\text { Rectem et al 2004) }\end{array}$ & Rappel différé : 15 & Normes \\
\hline & & Kectem et a1. 2004) & Pourcentage d'apprentissage : $66 \%$ & Normes \\
\hline & Mémoire Visuelle & Figure Complexe de Rey (1959) & $\begin{array}{c}\text { Rappel Immédiat : } 22 \text { (Pc30-50); } \\
\text { Rappel Différé : } 22 ; \operatorname{Pc} 30-50)\end{array}$ & Normes \\
\hline \multirow{7}{*}{$\begin{array}{l}\text { Fonctions } \\
\text { executives }\end{array}$} & Contrôle inh ibiteur & $3^{\text {ème }}$ partie du Stroop Test (Delis \& Kaplan) & $42^{\prime \prime} ; \mathrm{NS} 12$ & Normes \\
\hline & Planification & $\begin{array}{l}\text { Test des Comissions, modifiés } \\
\text { (Pelletier et al, 2009) }\end{array}$ & $\begin{array}{c}\text { Temps de planification : } 5^{\prime} 30 ; \text { Pc50- } \\
70)\end{array}$ & Normes \\
\hline & & & Erreur totale : $0 ;$ Pc $80-95$ & Normes \\
\hline & & Trois Frises & en ordre & Normes \\
\hline & Fluence Visuo- & Test des 5 points (Tucha et al, 2012) & $31 ; \mathrm{Pc} 10-15) / / 1$ répétition $(3,2 \%)$ & Normes \\
\hline & Flexib ilité mentale & 4ème partie du Stropp (Delis \& Kaplan) & $37^{\prime \prime} ; \mathrm{NS} 14 ; 1$ erreur NC & Normes \\
\hline & & Partie B du Trail-Making Test (GREFEX, 2008) & $\begin{array}{c}\text { 51", Pc50-75, } 0 \text { erreur } \\
\text { B-A }=35 ", \text { Pc } 25\end{array}$ & Normes \\
\hline \multirow{3}{*}{$\begin{array}{l}\text { Fonctions } \\
\text { attentionnelles }\end{array}$} & $\begin{array}{l}\text { Vitesse de traitement } \\
\text { Attention divisée }\end{array}$ & Partie A du Trail-Making T est (GREFEX, & $\begin{array}{c}16 ', \text { Pc95, } 0 \text { erreur } \\
\text { Modalité visuelle : médiane } 733 \\
(\mathrm{Pc} 54)-\mathrm{ET} 113(\mathrm{Pc} 88)-\mathrm{Om} 1(\mathrm{Pc} 42)\end{array}$ & Normes \\
\hline & & $\begin{array}{l}\text { TAP, Tests d'évaluation de l'attention, } \\
\text { version 2.3.1 ( } \mathrm{Z} \text { immerman \& Fimm) }\end{array}$ & $\begin{array}{c}\text { Modalité aud itive : médiane } \\
730(\operatorname{Pc} 2)-\mathrm{ET} 183(\operatorname{Pc} 8)-\mathrm{Om} 1(\operatorname{Pc} 31)\end{array}$ & Déficitaire \\
\hline & A ttention sou tenue & $\begin{array}{l}\text { TAP, T ests d'évaluation de l'attention, } \\
\text { version 2.3.1 (Zimmerman \& Fimm) }\end{array}$ & $\begin{array}{c}\text { Nombe tota1 d'omisson : } 2 ; \mathrm{Pc} 24 \\
\text { stoppée après } 3 \text { minutes, car trop } \\
\text { dificile }\end{array}$ & $\begin{array}{c}\text { Normes } \\
\text { Déficitaire }\end{array}$ \\
\hline Raisonnement & Visuo-Spatial & $\begin{array}{l}\text { Matrice de l'échelle d'intelligence WAIS-IV } \\
\text { (Wechsler, 2008) }\end{array}$ & $25 ; \mathrm{NS} 15$ & Normes \\
\hline \multirow{3}{*}{ Questionnaires } & Humeur dépressive & $\begin{array}{c}\text { Beck Depression Inventory (BDI II; Beck, } \\
1998)\end{array}$ & 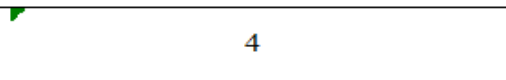 & Normes \\
\hline & Humeur anxieuse & $\begin{array}{c}\text { State Trait Inventory Anxiety (STAI A et B, } \\
\text { Spielberg, 1980) }\end{array}$ & $\begin{array}{c}\text { an xété trait : } 44 / 80 \text {; an xiété état : } \\
440 / 80\end{array}$ & Normes \\
\hline & Fatigue & Fatigue Impact Scale (FIS ; Fisk et al, 1994) & Score non significatif & Normes \\
\hline
\end{tabular}

Note. Sauf mention contraire, les normes sont celles des fournisseurs des tests. Elles sont établies selon l'âge, plus rarement selon le sexe et le niveau d'éducation. Rappelons que, du centile 100 au centile 96 (Note standard (NS) : $\geq 16$; score Z $\geq$ 1.75), la valeur est très supérieure à la norme, qu'elle est supérieure à la norme entre les centile 85 et 95 (NS : 14-15; score $\mathrm{Z}$ entre 1,05 et 1,65), dans la norme entre les centiles 16 et 84 (NS de 7 à 13 ; score $Z$ entre 1 et -1 ), qu'elle est dans les normes inférieures entre les centiles 6 et $15(\mathrm{NS}=6$; Score $\mathrm{Z}$ entre $<-1.05$ et $>-1.6)$, et est déficitaire, nettement à très inférieur à la norme, pour $\mathrm{Pc} \leq 5$ ((NS $\leq 5 ;$; Score $Z \leq-1.65)$. La moyenne est au centile $50(\mathrm{NS}=10$; Score $Z=0)$. Cette interprétation des résultats correspond aux lignes directrices établies par l'Association Suisse des Neuropsychologues, version 2018. Pour les tests ne possédant pas de normes pour la population de référence, une appréciation clinique est proposée. 